\title{
Age and sex differences in discrimination learning
}

\author{
MITRI E. SHANAB and FAITH H. McCLURE \\ California State University, Fresno, California
}

\begin{abstract}
The present study tested the hypothesis of a decreasing ontogenetic trend in the rate of learning a discrimination as a function of age. A three-phase optional shift design was employed. One hundred and twenty male and female subjects from three age groups, 50-59, 60-69, and 70 years and older, were tested. The results indicated that, with advancing age, more trials were required to meet the learning criterion in the initial and shift discrimination phases. Female subjects learned the initial discrimination significantly faster than did male subjects. Although subjects aged 70 years and older were less likely than those in the younger age groups to choose a reversal shift in the test phase, they chose the reversal and extradimensional shifts at comparable rates. The finding of a reversed ontogenetic trend as a function of age is discussed in terms of both the mediation and attention theories.
\end{abstract}

A number of investigations using children and young adults have shown that whereas preschool children learn an extradimensional (ED) shift more rapidly than a reversal (R) shift, older children and young adults learn the $\mathrm{R}$ shift more rapidly than the ED shift. Kindergarten children, on the other hand, learn both shifts at comparable rates (H. H. Kendler \& T. S. Kendler, 1975). Typically, the subject first learns to discriminate between two stimuli that differ on two dimensions, with one dimension arbitrarily designated as relevant and the other as irrelevant. In the shift, the previously relevant dimension remains relevant but the previously incorrect cue of the relevant dimension becomes correct. Thus, if the two dimensions were form (e.g., triangle or circle) and color (e.g., black or white), and circle was correct regardless of color, then triangle would become correct regardless of color in the $\mathrm{R}$ shift, and color, regardless of form, would become relevant in the extradimensional shift.

The mandatory design, as used initially, contained certain methodological problems, since subjects given the ED shift would be correct on half the trials if they continued to respond as they had during the initial phase. Several modifications have been introduced to control for this fortuitous partial reinforcement. One of these modifications, introduced by Buss (1956) as the optional shift design, allows subjects to solve the discrimination in the manner they wish (see Figure 1).

Following criterion learning on the initial discrimination, the subjects are presented with only one of the pairs of stimuli used earlier, with the reinforcement reversed. In this way, both dimensions are made redun-

This article is based on the second author's master's thesis. Reprint requests should be sent to: Faith H. McClure, Department of Psychology, University of California, Los Angeles, California 90024. The senior author, Mitri E. Shanab, passed away on July $20,1983$.

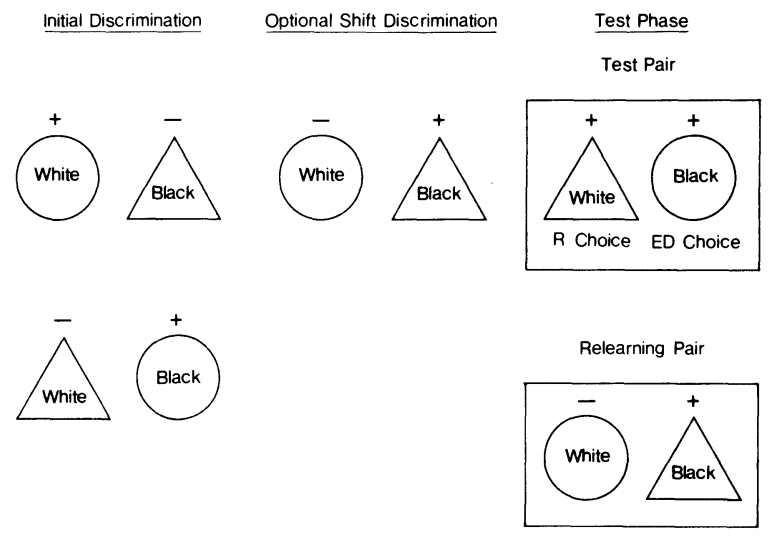

Figure 1. Illustration of the optional shift design.

dant and relevant. The subjects then have the choice of learning the shift by using either a $\mathrm{R}$ or an ED strategy. To test which strategy has been employed, a third phase is introduced in which the original Phase 1 stimulus pair is used and both stimuli are reinforced. Subjects who consistently choose the stimulus that was previously the negative cue of the relevant dimension are labeled reversal subjects, and those who choose the previously positive cue of the irrelevant dimension are labeled extradimensional subjects. To insure that the subjects are responding as they did in the previous phase, the stimulus pair used in that phase is interspersed with the test pair.

Research using both the mandatory and the optional shift designs has shown that the probability of making the $\mathrm{R}$ shift rather than the ED shift increases with age, at least within the preschool to college age range (T.S. Kendler \& H. H. Kendler, 1970). H.H. Kendler and T.S. Kendler (1962) proposed a two-stage mediation 
theory to explain this difference in shift behavior. According to this model, the behavior of young children is controlled by a single-unit S-R system (see Spence, 1936) according to which direct associations are formed between the external stimulus and the overt response, with no intervening links. As a result, young children find the ED shift easier to learn than the $\mathrm{R}$ shift because only one, rather than both, of the previously reinforced responses is replaced. Older children and young adults, however, behave according to a two-stage S-r-s-R system, in that they learn to discriminate by utilizing a conceptual mediating link (r-s) between the external stimulus and the overt response. Consequently, these subjects find the R shift easier than the ED shift because the same mediating response is used in the shift. The extradimensional shift is more difficult to execute because it requires the extinction of the old mediating response and the acquisition of a new mediating response. Although most of the support of the Kendlers' two-stage mediational theory is based on studies that used American subjects (H. H. Kendler \& T. S. Kendler, 1975), additional support has been reported in studies that used Jordanian subjects (Shanab \& Yasin, 1978, 1979).

Zeaman and House (1963) have proposed an alternative theory that assumes that discrimination learning involves two responses. The first response entails attending to a stimulus dimension, and the second involves an instrumental choice response to one of the stimulus cues. The probability of making a response to either of the two cues increases or decreases depending on whether or not the response in question is reinforced. The subject must thus attend to the relevant dimension (e.g., form) and approach the correct cue of that dimension (e.g., circle or triangle).

Support for Zeaman and House's (1963) attention theory comes from studies in which intradimensional shifts (ID) are compared with extradimensional shifts. This comparison is similar to the R-ED shift comparison, except that, unlike the latter comparison, in which the same stimuli are used throughout, stimuli with new values are used during the shift phase in the ID-ED shift comparison. The attention theory predicts that, regardless of age, the ID shift would be learned faster than the ED shift because the attention response learned during the initial discrimination transfers in an ID shift. This prediction did not receive support in at least two recent studies (T.S. Kendler \& H.H. Kendler, 1970; Shanab \& Yasin, 1979) in which significant interaction effects between age and type of shift were found, which indicates that whereas 4- and 6-year-old children solved both shifts at comparable rates, older children and young adults solved the ID shift faster than the ED shift and chose the ID shift more frequently than the ED shift.

The question of whether developmental changes in discrimination learning behavior observed in subjects between preschool and college age continue in older sub- jects has only recently been investigated (Coppinger \& Nehrke, 1972; Nehrke, 1973; Nehrke \& Coppinger, 1971). The results of the three studies showed that there was no significant difference among elderly subjects, aged 55 years and older, in terms of the rate of solving either the $R$ shift or the ED shift. Since the behavior of these subjects was similar to that observed in preschool children, Nehrke (1973) and Nehrke and Coppinger (1971) interpreted their findings as implying that these elderly subjects might be in transition from mediational sufficiency to mediational deficiency.

Shanab and Qassim (1978) examined more closely the effect of aging on discrimination learning in three groups of Jordanian subjects aged 50-59, 60-69, and 70 years and older, and found that subjects aged 50-59 years, similarly to older children and young adults, solved the $\mathrm{R}$ shift faster than the ED shift. However, those subjects aged 60-69 years, similarly to kindergarten children, solved the $\mathrm{R}$ and the ED shifts at comparable rates. Subjects aged 70 years and older, on the other hand, similarly to preschoolers, solved the ED shift faster than the R shift. Shanab and Qassim interpreted this finding of a reversed ontogenetic trend as suggesting that after middle age, subjects function according to a single-unit S-R system.

The purpose of this study was to investigate further the effect of age on the discrimination learning behavior of older adults in the United States by using the optional shift paradigm rather than the mandatory shift paradigm used in the Shanab and Qassim (1978) study. Based on the Coppinger and Nehrke (1972), Nehrke (1973), Nehrke and Coppinger (1971), and Shanab and Qassim (1978) investigations, it was hypothesized that a decreasing (or reversed) ontogenetic trend would be observed in the test phase, and that the rate at which the discriminations were learned in both the initial and the shift phases would be a decreasing function of age.

The present study also investigated discrimination learning as a function of sex. Young (1971) found that although men were superior to women in solving problems prior to age 60 years, they were inferior to women after that age. In contrast, Nehrke (1973) and Shanab and Qassim (1978) observed no sex-related differences. Since this investigation used tasks similar to those used in the Nehrke (1973) and Shanab and Qassim (1978) studies, it was expected that no sex-related differences would be found.

\section{METHOD}

\section{Design}

A $3 \times 2$ three-phase factorial design combining sex and three age groups $(50-59,60-69$, and 70 years and older) was used. In Phase 1 , the subjects learned to choose the correct member of a pair of stimuli that differed on two dimensions: shape (circle or triangle) and color (black or white). In Phase 2, the subjects were presented with only one of the stimulus pairs used in Phase 1, 
with the reinforcement contingency reversed. In Phase 3 , both stimulus pairs were presented. For the test pair (the pair presented in Phase 1 but not in Phase 2), both values were reinforced. The reinforcement contingency for the relearning pair (the pair given in Phase 2 ) remained the same.

\section{Subjects}

The subjects were 120 male and female volunteers. There were 19 males and 21 females in each age group. Each subject was either a member of a church or a community service organization or a resident of a privately owned senior citizens' complex. All subjects had received some formal schooling and were literate.

\section{Apparatus}

The apparatus consisted of an overhead projector used to present the stimuli. The stimuli were imposed on eight transparencies and were divided into two sets, A and B. For each set, there were four possible combinations of shape (circle or triangle), color (black or white), and position (left or right). Set A used only white circles and black triangles, and Set B used only white triangles and black circles in Phase 2. Although all four combinations were used in Phases 1 and 3, only two of the combinations were used in Phase 2.

\section{Procedure}

The experimenter told each subject that she was interested in seeing how people solve certain problems. The subjects were told that their task was to guess which of two shapes, circle or triangle, which could be either black or white, was correct. They were also told that if they tried hard enough, they would be able to make the correct choice every time.

The subjects were tested individually and were seated in front of a white screen; the experimenter was positioned behind the subjects to operate the projector.

The experiment was run in three phases, with order of presentation, positive stimulus cue, and position counterbalanced. The subjects were given feedback after each choice in each phase, with the experimenter saying "right" to a correct response and "wrong" to an incorrect response. The stimuli were presented one pair at a time for $5 \mathrm{sec}$. If no response was made in that time period, the stimulus pair was removed and presented again after a short delay. On each presentation, the experimenter asked the subject to guess which member of the pair was the correct one.

In Phase 1, the subjects were required to learn which member of the stimulus pair was correct. The subjects were trained to a criterion of 10 consecutive correct responses or were given 100 trials. None of the subjects failed to reach criterion within these limits.

In Phase 2, which followed Phase 1 immediately, the subjects were presented with only one of the stimulus pairs used in Phase 1 and the reinforcement contingencies were reversed (see Figure 1). The criterion for learning was 10 consecutive correct choices. All subjects reached criterion within 50 trials.

During Phase 3, which was started immediately after completion of Phase 2, a test pair, which was the same stimulus pair presented in Phase 1 but not in Phase 2, was presented to all subjects; responses to both cues were correct. The stimulus pair used in Phase 2 (the relearning pair) was interspersed randomly with the test pair, with its reward contingency unchanged. The test pair and the relearning pair were presented 10 times each. Each time the subjects made an incorrect response when the relearning pair was presented, the pair was presented again until the subjects made the correct choice. The relearning pair was used as a safeguard to ensure that the subjects continued to respond in the test phase by using the same strategy as in the previous shift phase.

The subjects who chose at least 8 out of 10 times the stimulus that was previously the negative cue of the relevant dimension were labeled reversal subjects, and those who chose at least
8 out of 10 times the previously positive cue of the irrelevant dimension were labeled extradimensional subjects.

\section{RESULTS}

The results of a two-way analysis of variance performed on the mean number of trials needed to learn the initial discrimination yielded significant main effects for age $[F(2,114)=5.01, p<.01]$ and $\operatorname{sex}[F(1,114)=4.75$, $\mathrm{p}<.05]$. No significant age $x$ sex interaction effect was obtained $(F<1)$. Although female subjects learned the task significantly faster than male subjects, the results of a Newman-Keuls test $(p<.05)$ revealed that, regardless of sex, subjects who were 50-59 years old learned the task significantly faster (mean $=14.27$ trials) than those who were 60-69 years old (mean $=21.14$ trials) and 70 years and older ( mean $=23.63$ trials). The means of the last two groups did not differ significantly from each other.

The results of a two-way analysis of variance performed on the mean number of trials required to learn the discrimination task in Phase 2 yielded a significant main effect for age $[F(2,114)=4.70, p<.025]$. Neither the effect of sex nor the interaction of age and sex was significant (both $F s<1$ ). The results of the NewmanKeuls comparison $(p<.05)$ showed that the oldest subjects learned the discrimination significantly slower than those in the two younger age groups, which did not differ significantly from each other. The means for the subjects who were 70 years and older, 60-69 years old, and $50-59$ years old were $17.33,14.25$, and 13.47 trials, respectively.

Choice of the reversal shift was a decreasing function of age, in that of 40 subjects in each age group, the number of reversal subjects in the 50-59-years-old, 60-69years-old, and 70-years-and-older age groups was 32, 28, and 20 , respectively. No significant difference between the male and female subjects was obtained $\left[\chi^{2}(1)<1\right]$. Collapsed across the sex variable, the result of the analysis of the reversal data as a function of age and type of shift yielded a significant effect $\left[\chi^{2}(2)=8.39\right.$, p $<$ $.02]$, indicating that, with increasing age, fewer subjects chose the reversal shift. To supplement the chi-square test, a 3 (age) x 2 (sex) analysis of variance was performed on the mean number of reversal choices. A significant main effect for age $[\mathrm{F}(2,114)=4.43, \mathrm{p}<$ .025] was obtained. Neither the sex factor $[F(1,114)=$ 1.33] nor the interaction of age with $\operatorname{sex}(F<1)$ yielded significant results. Newman-Keuls comparisons $(p<.05)$ showed that, whereas subjects in the 50-59- and 60-69years-old age groups did not differ significantly from each other with respect to the number of reversal choices, they did differ significantly from those in the 70-yearsand-older age group.

\section{DISCUSSION}

The main finding of the present study indicates that after age 50 years, subjects apparently resort less frequently to using the 
reversal than to using the extradimensional shift strategy in solving discrimination problems. This finding confirms and extends the findings of Coppinger and Nehrke (1972), Nehrke (1973), Nehrke and Coppinger (1971), and Shanab and Qassim (1978), which suggest an ontogeny of shift behavior in older adults that is a reversal of the ontogeny found in children between preschool and college age.

In the present study, a transition period was observed in the 70-years-and older age group, in that they were equally likely to choose either a reversal of an extradimensional shift. These findings are similar to those reported by Coppinger and Nehrke (1972), Nehrke (1973), and Nehrke and Coppinger (1971), whose subjects aged 55 years and older solved the reversal and extradimensional shifts at comparable rates. Shanab and Qassim (1978), however, observed the transition period in their 60-69years-old age group. This transitional stage in development has been interpreted by T.S. Kendler and H. H. Kendler (1959) as resulting from the fact that some subjects use a conceptual mediating link between the external stimulus and the overt response, whereas others operate according to a single-unit S-R system. Nehrke and his associates proposed that the transition period in the older age group indicated a transition from mediational sufficiency to mediational deficiency. This might also explain the inferior performance of the older subjects in both the initial and shift discrimination phases in the present study.

It is possible that the inferior performance of the oldest group reflects a decline in their attentional capacity. In reviewing the literature on encoding operations, Hasher and Zacks (1979) concluded that elderly subjects exhibit a reduction in their ability to effortfully process information. As a result, they are not as proficient as young subjects in performing tasks that require attentional effort. The inferior performance of the oldest group may have also been influenced by a decline in their ability to discriminate relevant from irrelevant features of the environment (Hoyer, Rebok, \& Sved, 1979; Rabbit, 1977) or by a decline in their ability to classify and categorize information (Cicirelli, 1976; Denney, 1974).

An interesting and unexpected finding in the present investigation was that the female subjects solved the initial discrimination significantly faster than the male subjects. These results are inconsistent with Nehrke's (1973) and Shanab and Qassim's (1978) findings. Several variables may account for the discrepancy. For example, the Shanab and Qassim study differs from the present one in that different experimental designs were used. Furthermore, the subjects in the two studies differed with regard to cultural and educational backgrounds. The Nehrke study also differed from the present study in terms of the experimental design and age groups employed.

The superiority of the females relative to the males in solving the initial discrimination is more in line with Young's (1971) findings. Young tested 40 married couples aged 41 to 76 years on various problem solving tasks and found an age $\mathrm{x}$ sex interaction. Specifically, she found that before age 60 years, the males were superior to the females in solving some of the problems. After age 60 years, these sex-related differences diminished and the performance of the females was slightly, although insignificantly, superior to that of the males. Close examination of the data in both Young's study and the present study indicates that the performance of the females changed very little with increasing age, whereas the performance of the males was adversely affected by increasing age. Further research examining the effect of sex on problem solving behavior in the elderly needs to be carried out.
In conclusion, the present study provides additional evidence for a reversed ontogenetic trend as a function of age and suggests that the developmental changes in older adults occur regardless of diverse cultural differences or marked variations in experimental design.

\section{REFERENCES}

Buss, A. H. Reversal and nonreversal shifts in concept formation with partial reinforcement eliminated. Journal of Experimental Psychology, 1956, 52, 162-166.

Cicirelli, V. G. Categorization behavior in aging subjects. Journal of Gerontology, 1976, 31, 676-680.

Coppinger, N. W., \& Nehrke, M. F. Discrimination learning and transfer of training in the aged. Journal of Genetic Psychology, 1972, 120, 93-102.

Denney, N. W. Classification abilities in the elderly. Journal of Gerontology, 1974, 29, 309-314.

HASher, L., \& ZACKs, R. T. Automatic and effortful processes in memory. Journal of Experimental Psychology: General, 1979, 108, 356-388.

Hoyer, W. J., Reвoк, G. W., \& Sved, S. M. Effects of varying irrelevant information on adult age differences in problem solving. Journal of Gerontology, 1979, 34, 553-560.

KENDLER, H. H., \& KendleR, T. S. Vertical and horizontal processes in problem solving. Psychological Review, 1962, 69, 1-16.

Kendler, H. H., \& KendleR, T. S. From discrimination learning to cognitive development: A neobehavioristic odyssey. In W. K. Estes (Ed.), Handbook of learning and cognitive processes (Vol. 1). Hillsdale, N.J: Erlbaum, 1975.

Kendler, T. S., \& Kendler, H. H. Reversal and nonreversal shifts in kindergarten children. Journal of Experimental Psychology, 1959, 58, 56-60.

Kendle R, T. S., \& KendleR, H. H. An ontogeny of optional shift behavior. Child Development, 1970, 41, 1-27.

Nehrke, M. F. Age and sex differences in discrimination learning and transfer of training. Journal of Gerontology, 1973, 28, 320-327.

Nehrke, M. F., \& Coppinger, N. W. The effect of task dimensionality on discrimination learning and transfer of training in the aged. Journal of Gerontology, 1971, 26, 151-156.

RABBITT, P. Changes in problem solving abilities in old age. In J. E. Birren \& K. W. Schaie (Eds.), Handbook of the psychology of aging. New York: Van Nostrand Reinhold, 1977.

ShanAB, M. E., \& QAssim, K. A. The effect of aging on reversal and extra-dimensional shifts in Jordan. Journal of Cross-Cultural Psychology, 1978, 9, 339-347.

Shanab, M. E., \& Yasin, A. B. A comparison of reversal and extradimensional shifts in Jordanian children. Bulletin of the Psychonomic Society, 1978, 11, 109-111.

Shanab, M. E., \& Yasin, A. B. Intradimensional and extradimensional shifts by Jordanian students. Journal of General Psychology, 1979, 100, 199-213.

SPEnCE, K. The nature of discrimination learning in animals. Psychological Review, 1936, 43, 427-449.

Young, M. L. Age and sex differences in problem solving. Journal of Gerontology, 1971, 26, 330-336.

Zeaman, D., \& House, B. J. The role of attention in retardate discrimination learning. In N. R. Ellis (Ed.), Handbook of mental deficiency. New York: McGraw-Hill, 1963.

(Manuscript received for publication May 14, 1983.) 